\title{
Delayed surgical and non-surgical treatment of
} placental remnants: no difference was found in the clinical efficacy and long-term pregnancy outcomes

This article was published in the following Dove Press journal:

Therapeutics and Clinical Risk Management

\section{Weilin Chen \\ Zhibo Zhang \\ Xinyan Liu}

Department of Obstetrics and Gynaecology, Peking Union Medical College Hospital, Beijing, People's Republic of China
Correspondence: Xinyan Liu Department of Obstetrics and Gynaecology, Peking Union Medical College Hospital, No I Shuaifuyuan, Dongcheng District, Beijing 100730 , People's Republic of China

$\mathrm{Tel}+8618612671860$

Fax +861065124875

Email liuxinyan_pumch@126.com
Purpose: In a tertiary hospital, patients facing delayed treatment with placental remnants were common. The aim of this study was to assess the clinical efficacy and long-term pregnancy outcomes of the delayed surgical/non-surgical treatment for placental remnants.

Patients and methods: The records of referral patients with placental remnants after second/ third-trimester delivery/termination of pregnancy were retrospectively analyzed. A long-term follow-up was made by phone to inquire about the future pregnancy outcomes. The measurements of clinical efficacy included the postpartum intervals for Doppler ultrasound to become normal and for menstrual cycle to return to normal. Conception rate, interval for future pregnancy and obstetric outcomes were used to assess future pregnancies.

Results: A total of 65 patients, who were clinically diagnosed with placental remnants after termination of pregnancy at the second or third trimester from 2000 to 2016, were included in this study. Delayed surgical treatments employed at a median interval (MI) of 2.7 months after termination of pregnancy had a similar interval for ultrasound $(P=0.353)$ and menstrual cycle $(P=0.751)$ to return to normal compared with non-surgical treatments. For non-lactating patients who accepted expectant treatments, the postpartum interval for ultrasound to return to normal was significantly longer than that for menstrual cycle to become normal ( $\mathrm{MI}=3.6,1.5$ months, respectively, $P=0.000)$. For all of the patients successfully treated, the conception rate $(P=1.00)$, the interval for a second pregnancy $(P=0.771)$, ongoing-pregnancy/live birth rate $(P=0.419)$ and the recurrence rate of placenta accrete $(P=1.00)$ there was no significant difference between non-surgical and surgical treatments. Assisted selective uterine artery embolization at an MI of 23 days after the termination of pregnancy had a longer interval for ultrasound to become normal than single expectant treatment $(P=0.017)$. For all patients after expectant treatments, $94.1 \%$ of patients conceived after an MI of 12.5 months with the ongoing-pregnancy/live birth rate of $66.7 \%$ and a $33.3 \%$ recurrence rate of placenta accreta.

Conclusion: According to our experience, delayed surgeries at a postpartum interval of 2.7 months might have similar clinical efficacy and long-term pregnancy outcomes with expectant treatment in treating placental remnants.

Keywords: placental remnants, clinical efficacy, reproductive outcome, conservative treatment, surgical treatment, placental remains

\section{Introduction}

Placental remnants can be found after miscarriage, induction of labor, transvaginal delivery or cesarean section. ${ }^{1-3}$ Broadly defined placenta accreta, which included placenta increta and percreta, was one of the main causes of placental remnants. ${ }^{4}$ The conservative treatment of placenta accreta, which preserves the uterus and 
leaves the placenta adhered partially or totally in situ, with or without the assistance of methotrexate or uterine artery embolization (UAE) treatment, could lead to iatrogenic placental remnants. ${ }^{5,6}$

The diagnosis of placental remnants is still a clinical challenge. ${ }^{3,7}$ Currently, there is no consensus on the treatment of placental remnants after delivery. ${ }^{2-4}$ Interventions performed at least $24 \mathrm{~h}$ after delivery were defined as delayed surgical interventions, which increase the risk of complications. ${ }^{2}$ However, in some cases, the diagnosis was made after delivery, and the patients had to accept the delayed interventions, especially in referral hospitals where the referred patients could be several weeks or even months after delivery or termination of pregnancy. ${ }^{7}$ Treatment priorities in these patients are unknown and need to be learned.

Clinically applied surgical treatments included dilatation and curettage (D\&C) and hysteroscopy or laparoscopy (H/L) to remove the placental tissue. The non-surgical treatment of placental remnants commonly resembled the conservative treatment experience of placenta accreta, such as expectant treatment (assisted by selective UAE or not), which has been increasingly reported chronologically. ${ }^{1}$

During the past 3 decades, the incidence of placenta accreta has obviously increased mainly due to the increased rate of cesarean delivery. ${ }^{8-10}$ In China, as the two-child policy was implemented in 2016, the patients diagnosed with placenta accreta would select the treatment that could conserve their reproductive ability instead of peripartum hysterectomy. The incidence of placental remnants would also increase along with the increase in placenta accreta. Therefore, it is important to study the clinical efficacy of the delayed treatment of placental remnants and its effect on future pregnancies. However, there were limited studies regarding this topic, especially the outcomes of delayed treatments in the referral hospitals. ${ }^{7,11}$ This study retrospectively focused on the outcomes of the referral patients clinically diagnosed with placental remnants after termination of pregnancy at the second or third trimester. The clinical efficacy was compared between delayed surgical treatment and nonsurgical treatment. The long-term reproductive outcome was also analyzed for patients accepting different treatments.

\section{Patients and methods}

This was a retrospective cohort study. The medical records of the patients who were clinically diagnosed with placental remnants after termination of pregnancy at the second or third trimester from 2000 to 2016 in Peking Union Medical College Hospital (a tertiary referral teaching hospital) were reviewed. All of these patients were referral patients who had initial termination of pregnancy or delivery in other hospitals, and thus the treatments in our hospital were delayed treatments of placental remnants. The patients clinically diagnosed with placental remnants included: 1) during the delivery: had indications of placenta accreta or incomplete removal of the placenta and 2) postpartum follow-up: who complained of vaginal bleeding, abdominal pain, or fever. Imaging examinations such as Doppler ultrasound or magnetic resonance imaging (MRI) indicated placenta-like tissue inside the uterine cavity. The involved patient must accept non-medicine delayed treatment, including surgical and non-surgical treatments. Non-surgical treatment was mainly expectant treatment (await spontaneous expulsion of the placental tissue without application of medicine). Selective UAE was employed in some patients to assist the expectant process. The indications for selective UAE included: 1) an unsatisfactory decrease of $\beta$ human chorionic gonadotropin ( $\beta \mathrm{HCG}), 2$ ) a plateau was reached for $\beta \mathrm{HCG}$ level, and 3$)$ the intrauterine tissue had remarkable vascularity although the level of $\beta \mathrm{HCG}$ had become normal. Emergent angiographic selective embolization in cases of postpartum hemorrhage was not involved. Surgical procedures involved D\&C and H/L to remove the placental tissue. Patients diagnosed with cesarean scar pregnancy were excluded from this study because these patients tended to have morbid placental accreta and should be discussed separately. The patients who had placenta intentionally left inside their uterine cavity due to bleeding during their delivery, were also not included in this study. The ethics committee of the Chinese Academy of Medical Sciences approved this study, and written informed consent was obtained from each patient for using their data and medical records in research.

\section{Clinical efficacy}

In the short-term follow-up, the patients' symptoms were carefully observed. If the $\beta \mathrm{HCG}$ level was elevated for the patient, it was tested every week until normal. The transvaginal color Doppler ultrasound was performed every month until the placental remnants were completely cleared. If the patient had abnormal vaginal bleeding, infection indicated by fever, abdominal pain or tissue discharged from vagina, the patient came to the clinic at any time. The ultrasound mainly measured the length, thickness and width of the residual placental tissue (the median diameter of the tissue was defined as an average of the three abovementioned diameters), the blood flow signal around the tissue (without blood stream, with minimal to moderate amount vascularity or with 
marked vascularity compared to that in the myometrium) and whether the myometrium was thinner (defined as thinner compared with the myometrium around the placental lesion) or inseparable with the placenta tissue. Cure was defined as complete exclusion of the residual placental tissue indicated by the Doppler ultrasound.

The primary measurements assessing clinical efficacy were the postpartum interval for color Doppler ultrasound to become normal in the patients who were successfully treated by only one way of surgical/non-surgical treatments, and for patients who had termination of pregnancy at the second trimester, the postpartum interval for menstrual cycle to return to normal was also calculated. Patients with termination of pregnancy during the third trimester were not included because of postpartum lactation.

\section{Long-term pregnancy outcomes}

A long-term follow-up was made by phone annually inquiring about future pregnancy outcomes. The patient answered the following questions in the phone follow-up: whether the patient desired another pregnancy after the delivery/termination of pregnancy, how many times pregnancies were conceived and the corresponding year and outcomes (miscarriage/ongoing pregnancy/live birth). Each patient gave an oral consent for publishing her data. The follow-up data have been updated to June 2017. Conception rate, the interval for future pregnancy and the obstetric outcome were the main indicators to evaluate future pregnancies.

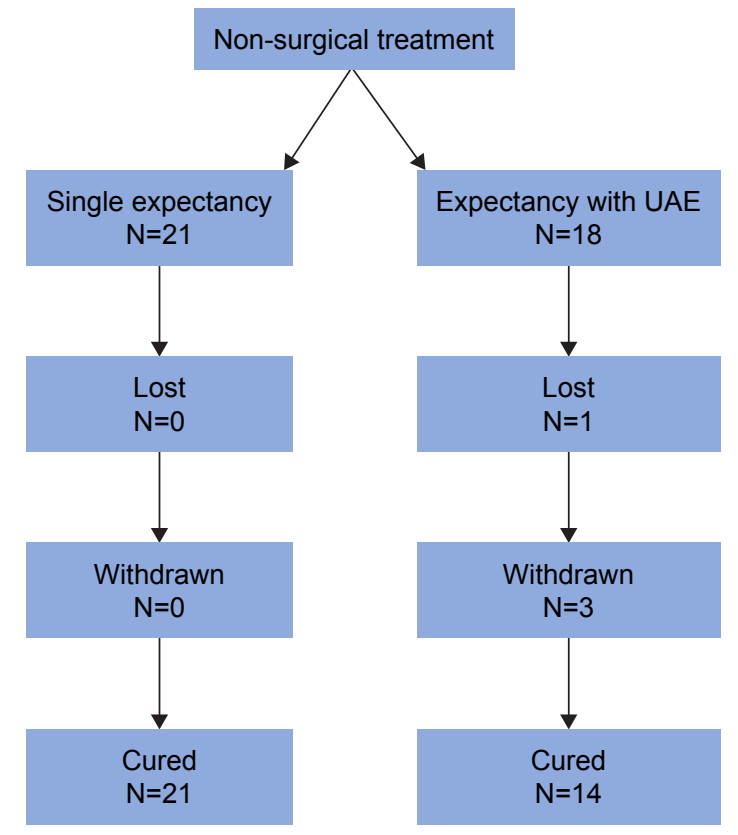

\section{Statistics}

The statistical analyses were performed using the SPSS 12.0 software programs (SPSS Inc., Chicago, IL, USA). Student's $t$-tests, Mann-Whitney tests, $\chi^{2}$ tests, Fisher's exact tests and Logistic regression model were utilized when required. Analysis of covariance was applied to control confounding factors while comparing quantitative data. Non-normal distribution data have been transformed with logarithmic function when necessary.

\section{Results}

Sixty-five patients met with the inclusion criteria, 39 of whom received non-surgical treatment, including 21 patients with single expectant treatment and 18 patients with expectant treatment assisted with UAE. The other patients had surgical treatment, 18 of them had D\&C and 8 patients had H/L operations. Figure 1 shows the clinical outcome for patients after non-surgical or surgical treatments. All of the patients with single expectant treatment finally successfully evacuated their intrauterine tissue and were included in the final analysis. Three patients (one had expectant treatment assisted with UAE and two accepted D\&C treatment) were lost in the short-term follow-up. They have not had their ultrasound followed until normal and were not successfully treated. Another three patients who initially accepted expectant treatment assisted with UAE were withdrawn because they required surgery before the placental remnants were completely cleared under ultrasound. These six patients

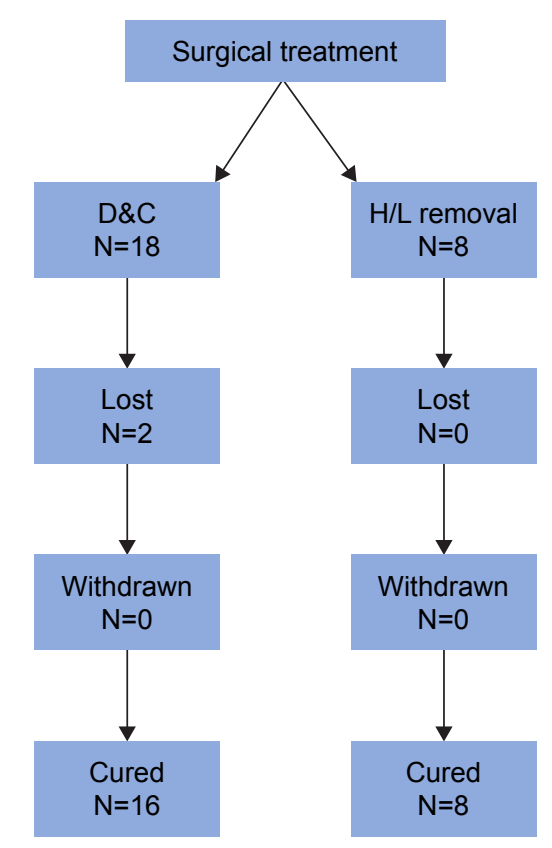

Figure I The diagram of the clinical outcomes of the included patients.

Abbreviations: D\&C, dilatation and curettage; H/L, hysteroscopy or laparoscopy; UAE, uterine artery embolization. 
Table I Clinical features of all the patients with placental remnants after delivery/termination of pregnancy at the second or third trimester

\begin{tabular}{ll}
\hline Features & \\
\hline General & $29.4 \pm 4.8$ \\
Age (years), mean \pm SD & $2(2-4)$ \\
Gestation (times), median (interquartile range) & $0(0-1)$ \\
Parity (times), median (interquartile range) & $43(66.2)$ \\
Number of patients who had past operation & \\
of uterine cavity, $\mathrm{n}(\%)$ & \\
Pregnancy related & \\
Gestational age & $50(76.9)$ \\
$\quad$ I2-28 weeks, $\mathrm{n}(\%)$ & 15 \\
$\quad 28-40$ weeks, $\mathrm{n}$ & \\
Way of delivery, $\mathrm{n}$ & 60 \\
$\quad$ Vaginal delivery & 5 \\
$\quad$ Cesarean delivery & \\
Reasons for termination at the second trimester & \\
Fetal deformity & 5 \\
Fetal chromosome abnormality & $\mathrm{I}$ \\
Intrauterine fetal death & 6 \\
Preterm premature rupture of membrane & 5 \\
Patient's required induced abortion & 22 \\
Severe comorbidity & $\mathrm{I}$ \\
Late abortion & 10 \\
Symptoms & \\
Vaginal bleeding, $\mathrm{n}$ (\%) & \\
Fever, $\mathrm{n}$ & $45(69.2)$ \\
Abdominal pain, $\mathrm{n}$ & 9 \\
None, $\mathrm{n}$ & 16 \\
\hline
\end{tabular}

were excluded in the analysis of clinical efficacy because they were neither entirely successfully treated nor successfully treated by one single treatment. The clinical features of the 65 involved patients are shown in Table 1. Before treatment, nine patients with fever (six patients in nonsurgical treatment and three patients in surgical treatment) were diagnosed with infections, which were all successfully controlled by antibiotics. During the treatment, there was no patient diagnosed with infection.

\section{Clinical efficacy}

The clinical efficacy of delayed surgical/non-surgical treatment of placental remnants is demonstrated in Table 2. It was shown that age and the postpartum interval between delivery/terminations of pregnancy to intervention were significantly different between the two groups. After controlling for age and the postpartum interval before intervention by analysis of covariates, it was detected that the postpartum interval for ultrasound $(P=0.353)$ to become normal had no significant difference between patients accepting delayed surgical and non-surgical treatments. These results indicated that delayed surgical treatments applied at a median interval (MI) of 76 days (2.7 months) would not accelerate the clearance of intrauterine placental remnants.

For the patients who accepted non-surgical treatments (whether assisted by UAE) and who had termination of pregnancy at the second trimester (patients without lactation) ( $\mathrm{n}=23)$, the postpartum MI for Doppler ultrasound to become normal was 3.6 months, which was significantly longer than the postpartum MI of 1.5 months for menstrual cycle to become normal $(P=0.000)$.

Neither marked vascularity (odds ratio [OR] 1.054, 95\% CI 0.366-3.040) nor indication of myometrium invasion under Doppler (thinner myometrium or inseparable myometrium with the placenta tissue) (OR $0.356,95 \% \mathrm{CI}$ 0.122-1.038) was the risk factor for the placental remnants to exist for $>5$ months postpartum.

In order to explore the differences between four specific therapeutic methods, analysis of covariates with the least significant difference test was utilized to compare clinical efficacy (Table 3). It was shown that expectant treatment assisted with UAE had significantly longer interval for ultrasound to become normal compared with single expectant treatment (mean difference $0.263,95 \%$ CI $0.049-0.478$, and the original data have been transformed with logarithmic

Table 2 Clinical efficacy of different delayed treatments of placental remnants

\begin{tabular}{|c|c|c|c|}
\hline Measurements & $\begin{array}{l}\text { Non-surgical } \\
(n=35)\end{array}$ & $\begin{array}{l}\text { Surgical } \\
(n=24)\end{array}$ & P-value \\
\hline Age (years) & $30.7 \pm 4.3$ & $27.2 \pm 5.2$ & 0.009 \\
\hline Size of the placental remnant (median diameter, $\mathrm{cm}$ ) & $4.0 \pm 1.9$ & $4.0 \pm 1.7$ & 0.908 \\
\hline Marked vascularity under color Doppler & $57.1 \%(20)$ & $66.7 \%(16)$ & 0.462 \\
\hline Thinner myometrium or inseparable with myometrium & $45.7 \%(16)$ & $58.3 \%(14)$ & 0.342 \\
\hline Postpartum interval between delivery/termination of pregnancy to intervention (days) & $17(7,53)$ & $76(18,104)$ & 0.002 \\
\hline Interval for color Doppler ultrasound to became normal (months) & $5.0(3,6.5)$ & $4.8(2.4,6.4)$ & $0.353^{\mathrm{a}}$ \\
\hline Interval for menstruation to return normal (termination at second trimester) & $\mathrm{n}=23, \mathrm{I} .5(\mathrm{I}, 3)$ & $\mathrm{n}=22,2(\mathrm{I}, 4.3)$ & $0.75 \mathrm{I}^{\mathrm{a}}$ \\
\hline
\end{tabular}

Notes: ${ }^{a}$ Analysis of covariates was used to control age and the postpartum interval between delivery/termination of pregnancy to intervention. Mean \pm SD was used to describe normally distributed data and median (interquartile range) was used to describe non-normally distributed data. Significantly different data was shown in bold. 
Table 3 Comparisons of the clinical efficacy of single expectant treatment, expectant treatment assisted with UAE, D\&C and H/L treatments

\begin{tabular}{|c|c|c|c|c|}
\hline \multirow[t]{2}{*}{ Measurements } & \multicolumn{4}{|l|}{$P$} \\
\hline & $\begin{array}{l}\text { Single } \\
\text { expectant } \\
\text { treatment, } \\
\mathbf{n}=2 \text { I }\end{array}$ & $\begin{array}{l}\text { Expectant } \\
\text { treatment } \\
\text { with UAE, } \\
n=14\end{array}$ & $\begin{array}{l}D \& C, \\
n=16\end{array}$ & $\begin{array}{l}H / L \\
\text { treatments, } \\
n=8\end{array}$ \\
\hline Interval for color & I & 0.017 & 0.970 & 0.183 \\
\hline ultrasound to & 0.017 & I & 0.043 & 0.503 \\
\hline \multirow[t]{2}{*}{ became normal } & 0.183 & 0.503 & 0.182 & I \\
\hline & $\begin{array}{l}\text { Single } \\
\text { expectant } \\
\text { treatment, } \\
n=1 \text { I }\end{array}$ & $\begin{array}{l}\text { Expectant } \\
\text { treatment } \\
\text { with UAE, } \\
n=12\end{array}$ & $\begin{array}{l}D \& C, \\
n=15\end{array}$ & $\begin{array}{l}H / L \\
\text { treatments, } \\
n=7\end{array}$ \\
\hline Interval for & I & 0.225 & 0.631 & 0.276 \\
\hline menstruation to & 0.225 & I & $0.47 I$ & 0.981 \\
\hline return normal & 0.276 & 0.981 & 0.480 & I \\
\hline
\end{tabular}

Notes: Significantly different data was shown in bold. Each line shows the comparison result of different treatments. For example, the first line means: compared with the treatment method of single expectant treatment, expectant treatment with UAE was significantly different (with $P$ value of 0.017 ), while $D \& C$ and $H / L$ were comparable (with $P$ values of 0.97 and 0.183 , respectively).

Abbreviations: D\&C, dilatation and curettage; H/L, hysteroscopy or laparoscopy; UAE, uterine artery embolization.

function) and $\mathrm{D} \& \mathrm{C}$ treatment (mean difference $0.267,95 \%$ CI 0.009-0.526). The interval for menstruation to return to normal (after second-trimester pregnancy) showed no significant difference between different treatments. The MI between delivery/termination of pregnancy to determination of treatment for expectant treatment assisted with UAE and D\&C treatment was 23 and 56 days, respectively.

For the three patients who were lost to short-term follow-up and were not successfully treated, one of them was diagnosed with sterility in the long-term follow-up and another two patients had intrauterine adhesion, one of whom had miscarriage. However, for the other three patients who required surgery after initial treatment of expectant treatment assisted with UAE, two of them desired another conception and both of them successfully conceived after an MI of 19.5 months. One of them conceived twice during the follow-up.

\section{Long-term pregnancy outcomes}

The long-term follow-up inquiring about the outcomes of future conceptions was available for $83.1 \%(54 / 65)$ of the included patients. The median follow-up length for all patients was 37 months. As shown in Table 4, the MI between delivery/terminations of pregnancy and another conception was 16 months (14.6 months for the non-surgical group and 19 months for the surgical group, $P=0.429$ ). For the patients who were successfully treated after treatments, the conception

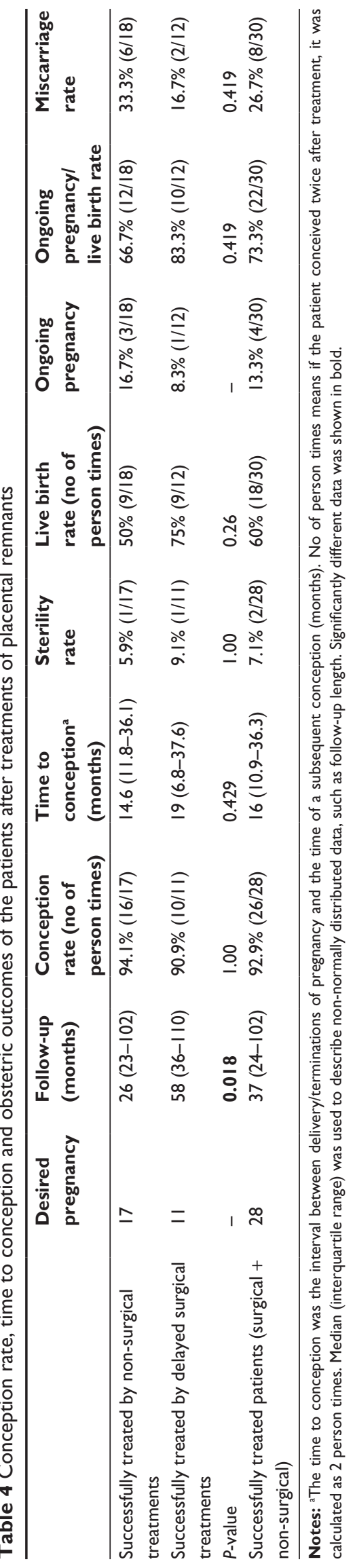


rate $(P=1.00)$, the interval for another conception $(P=0.771)$ and the ongoing pregnancy/live birth rate $(P=0.419)$ were all not significantly different between surgical and non-surgical treatments. All of the patients conceived naturally except one patient with in vitro fertilization due to polycystic ovary syndrome. The median follow-up length was actually shorter for patients after expectant treatments, mainly because as time went by, expectant treatment was increasingly employed at our department $(P=0.018)$. However, the time for another conception (from the cure of placental remnants to another conception) was 12.5 and 17.5 months for non-surgical and surgical groups, respectively, with no significant difference between groups ( $P=0.771$ ).

For the 18 patients who successfully delivered after their future pregnancy, four patients (two patients after non-surgical and two patients after surgical treatments) had placenta increta/percreta complicated with the diagnosis of postpartum hemorrhage. No patient accepted hysterectomy due to the placenta increta/percreta. Placenta accreta occurred in three (one patient after non-surgical and two patients after surgical treatments) patients, and one of them had a second time D\&C. The reoccurrence rate of broadly defined placenta accreta was not different between non-surgical $(3 / 9=33.3 \%)$ and surgical treatments $(4 / 9=44.4 \%)(P=1.00)$.

\section{Discussion}

\section{Surgical treatment}

There was no study comparing the clinical efficacy after different treatments of placental remnants, especially for patients who were facing delayed treatment. ${ }^{2}$ Traditionally, we suppose that surgical treatments directly remove tissue from the uterus and would accelerate the interval for Doppler ultrasound to return to normal and therefore improve the fertility and obstetric outcome. Whether this experience fits for the patients who were referred to a tertiary hospital and facing the treatment choice at a delayed time point was unknown. The results of this study indicated that delayed surgeries employed at an MI of 76 days (2.7 months) after the termination of pregnancy might neither accelerate the clearance of intrauterine placental remnants nor advance fertility or obstetric outcomes compared with non-surgical treatments, as the clinical efficacy and reproductive outcomes showed no difference between surgical and non-surgical treatments.

The results of this study indicated that, for referral patients who were already 2.7 months after delivery/termination of pregnancy, surgery might not be a priority as it would not benefit the patient in clinical efficacy or pregnancy outcome. The result of this study should remind the doctors in tertiary hospitals who are facing the referred patients with placental remnants that surgery might not be the first choice all the time. Attention should be paid that this result does not fit for all of the patients after surgical/non-surgical treatment of placental remnants because the patients we included were all referred patients who were facing delayed treatments (the MI in this study was 2.7 months). Whether surgical treatments performed at an earlier intervention time point would accelerate the process and benefit reproduction need to be verified in future studies.

\section{Expectant treatment (whether or not assisted by UAE)}

The efficacy and reproductive outcomes of expectant treatment in patients with placental remnants who required treatment 2.7 months after delivery/termination of pregnancy have been testified by this study.

\section{Clinical efficacy}

The postpartum interval for Doppler ultrasound to become normal was significant because as long as there was residual placental tissue inside the uterine cavity, the patient had the possibility of intrauterine infection, vaginal bleeding and abdominal pain. ${ }^{3}$ In the literature, for the patients preoperatively or intra-operatively diagnosed with placenta accreta and who had expectant treatment, it was reported that the median time for the placental tissue to be cleared was 3 months (1-5 months). ${ }^{6}$ In this study, we showed a median postpartum interval of 5 months for the patients who had placenta remnants and accepted expectant treatment, which was in accordance with the literature.

\section{Clinical course of the placental remnants}

The result of this study demonstrated that the postpartum MI (3.6 months) for Doppler ultrasound to become normal was significantly longer than that (1.5 months) for menstrual cycle to return to normal, in patients who accepted expectant treatment whether assisted by UAE or not $(P=0.000)$. This indicated that the clearance of the residual placental tissue was a slow progress, compared with the relatively faster progress for maternal menstrual cycle to return. On the other hand, it was shown that although the placental remnants were not completely cleared, the maternal menstrual cycle could return. In the literature, for the patients who accepted conservative treatment of placenta accreta in which the complete placenta was left in situ, the reported average time for menstrual cycle to return to normal was 4.5 months, and the placenta tissue was cleared at a median length of 9 months. ${ }^{12}$

Both the results of this study and that in the literature showed a clinical phenomenon of a slower anatomic regres- 
sion of placental tissue compared with the regression of hormone activity secreted by placenta. From these data, it could be seen that the postpartum follow-up for patients who had placental remnants was of great importance. After the menstrual cycle had returned to normal and the level of $\beta H C G$ had decreased to normal, it still took some time for the placental tissue to be completely excluded. The follow-up was essential to avoid complications and to distinguish diagnosis from other diseases such as trophoblastic diseases. ${ }^{13}$ Three of our patients who failed to finish the follow-up ended up with complication of intrauterine adhesion or sterility. Thus, we suggested patients to adhere to follow-up until the intrauterine tissue was entirely excluded and start to prepare for future pregnancy under doctor's advice.

\section{Assisted UAE in expectant treatment}

Although assisted UAE was repetitively reported to be effective in the conservative treatment of placental accreta, ${ }^{14,15}$ whether the method could improve the reabsorption of placental tissue still remained to be verified. It was shown in this study that after expectant treatment assisted with selective UAE at an MI of 23 days, the interval for ultrasound to become normal was significantly longer compared with single expectant treatment. This result was surprising because assisted selective UAE did not accelerate the clearance of placental remnants inside the uterine cavity, instead it prolonged the time to clear the placental remnants compared with simple expectant treatments. However, this was in accordance with the results from previous independent studies. ${ }^{6,16}$ It was mentioned earlier that the median time for placental tissue to be cleared was 3 months (1-5 m), ${ }^{6}$ whereas, if UAE was used as assistant treatment, the median time was reported to be 6 months $(1-12 \mathrm{~m}),{ }^{16}$ which seems to be longer than the expectant treatment.

Besides, assisted UAE might have complications such as post-embolism syndrome, inadvertent embolization of the external iliac or iliac arteries, leg ischemia and necrosis of the uterus or buttocks. ${ }^{16,17}$ Routine application of UAE and its indications was still under controversy. According to the result of this study, assisted selective UAE treatment for placental remnants undergoing expectant treatment, especially in patients without the indication of massive vaginal bleeding, should be employed with caution. ${ }^{16}$

\section{Long-term pregnancy outcomes}

Regarding reproductive outcome, it was traditionally thought that delayed treatment of placental remnants, especially expectant treatment, might increase the possibility of intrauterine adhesion and influence fertility. However, Barel et al ${ }^{18}$ verified that there was no correlation between the intrauterine adhesion rate and the interval from suspicion of placental remnants to treatment. ${ }^{18}$ The intrauterine adhesion was unable to be evaluated for each patient in this retrospective study. However, this study showed that the conception rate after expectant treatments of placental remnants could be as high as $94.1 \%$. Consistent with this study, the reported conception rate of conservative treatment of placenta accreta was $88 \%{ }^{19}$ The ongoing pregnancy/live birth rate in this study was as high as $71.4 \%$, which was comparable with the corresponding rate of $73.2 \%$ in the systematic review of literature. ${ }^{3}$

In Sentilhes et al's study, ${ }^{19} 131$ patients were retrospectively included, and the median time for another conception after conservative treatment of placenta accreta was 17.3 months, which was in accordance with the MI of 15 months in this study. Actually, most of the patients would not plan another pregnancy soon after the cure of placental remnants. The time the patients choose for another conception was mainly dependent on the time the gynecologist suggested as safe and appropriate for another conception. ${ }^{14}$ Besides, some patients would delay another conception by themselves worrying for another placenta accreta. This study reported that the reoccurrence rate of broadly defined placenta accreta was $33.3 \%$ after expectant treatment of placental remnants, which was in accordance with the reported rate of $22.8 \%-29 \%$ after conservative treatment of placenta accreta. ${ }^{5,7,19}$

\section{Limitations}

The result of this study need to be interpreted with caution, as the sample size was small and some of the important differences need to be confirmed in future studies. The patients included had potential heterogeneity for they ended pregnancies for different reasons (Table 1). However, it was believed that placenta was formed after the second trimester, and therefore, the etiology of placental remnants after the second and the third trimesters was believed to be similar, since both were after the formation of placenta. Therefore, the data of the second and third trimester were calculated together. Although an appropriate statistical method (analysis of covariance) was selected to control the confounding factors, the limitations of its retrospective nature could not be neglected. For example, the significant difference of age and the postpartum/interval from the termination of pregnancy to intervention could be well controlled in future cohort studies to reach a consistent baseline data and compare the effect of the treatment more directly. Many patients who had accepted treatment of methotrexate or misoprostol in the clinical course were excluded from this study, this was due to the controversy on the effect of medicine treatment for placenta accreta. ${ }^{16,20,21}$ The complications of different 
treatments, especially the incidence of intrauterine adhesion was unable to be evaluated for each patient, due to the retrospective nature. The high conception rate to some extent reflects the low intrauterine adhesion rate, as the intrauterine adhesion was always related to infertility. ${ }^{11}$ However, the post-treatment intrauterine adhesion should be evaluated by hysteroscopy in future studies.

\section{Conclusion}

The result of this study indicated that delayed surgeries performed at a postpartum interval of 2.7 months did not differ from expectant treatment with regard to the clinical efficacy and long-term pregnancy outcomes after treatment of placental remnants. The expectant treatment of placental remnants was feasible in selective referral patients who were facing delayed interventions, especially in referral hospitals. Further prospective studies are needed to verify the safety of expectant treatments in patients with placental remnants facing delayed interventions.

\section{Acknowledgment}

The authors are grateful to Tao Xu and Hongqiu Gu (epidemiologist and medical statisticians) for their help with statistical analysis.

\section{Disclosure}

The authors report no conflicts of interest in this work.

\section{References}

1. Hatfield JL, Brumsted JR, Cooper BC. Conservative treatment of placenta accreta. J Minim Invasive Gynecol. 2006;13(6):510-513.

2. Hooker AB, Muller LT, Paternotte E, Thurkow AL. Immediate and long-term complications of delayed surgical management in the postpartum period: a retrospective analysis. J Matern Fetal Neonatal Med. 2015;28(16):1884-1889.

3. Hooker AB, Aydin H, Brolmann HA, Huirne JA. Long-term complications and reproductive outcome after the management of retained products of conception: a systematic review. Fertil Steril. 2016;105(1): 156-61.e1-.e2.

4. Guarino A, Di Benedetto L, Assorgi C, Rocca A, Caserta D. Conservative and timely treatment in retained products of conception: a case report of placenta accreta retention. Int J Clin Exp Pathol. 2015;8(10): $13625-13629$.
5. Perez-Delboy A, Wright JD. Surgical management of placenta accreta: to leave or remove the placenta? BJOG. 2014;121(2):163-169. discussion $169-170$.

6. Sentilhes L, Ambroselli C, Kayem G, et al. Maternal outcome after conservative treatment of placenta accreta. Obstet Gynecol. 2010; 115(3):526-534.

7. Kabiri D, Hants Y, Shanwetter N, et al. Outcomes of subsequent pregnancies after conservative treatment for placenta accreta. Int $J$ Gynaecol Obstet. 2014;127(2):206-210.

8. Publications Committee, Society for Maternal-Fetal Medicine, Belfort MA. Placenta accreta. Am J Obstet Gynecol. 2010;203(5): $430-439$.

9. Zhong L, Chen D, Zhong M, He Y, Su C. Management of patients with placenta accreta in association with fever following vaginal delivery. Medicine (Baltimore). 2017;96(10):e6279.

10. Legendre G, Zoulovits FJ, Kinn J, Senthiles L, Fernandez H. Conservative management of placenta accreta: hysteroscopic resection of retained tissues. J Minim Invasive Gynecol. 2014;21(5):910-913.

11. Ben-Ami I, Melcer Y, Smorgick N, Schneider D, Pansky M, Halperin R. A comparison of reproductive outcomes following hysteroscopic management versus dilatation and curettage of retained products of conception. Int J Gynaecol Obstet. 2014;127(1):86-89.

12. Torrenga B, Huirne JA, Bolte AC, van Waesberghe JH, de Vries JI. Postpartum monitoring of retained placenta. Two cases of abnormally adherent placenta. Acta Obstet Gynecol Scand. 2013;92(4):472-475.

13. Hovav Y, Almagor M, Golomb E, Beller U. Intraplacental choriocarcinoma in residual placenta 8 months postpartum. Eur J Obstet Gynecol Reprod Biol. 2014;176:199-200.

14. Salomon LJ, deTayrac R, Castaigne-Meary V, et al. Fertility and pregnancy outcome following pelvic arterial embolization for severe post-partum haemorrhage. A cohort study. Hum Reprod. 2003;18(4): 849-852.

15. Sivan E, Spira M, Achiron R, et al. Prophylactic pelvic artery catheterization and embolization in women with placenta accreta: can it prevent cesarean hysterectomy? Am J Perinatol. 2010;27(6):455-461.

16. Fox KA, Shamshirsaz AA, Carusi D, et al. Conservative management of morbidly adherent placenta: expert review. Am J Obstet Gynecol. 2015;213(6):755-760.

17. Gizzo S, Saccardi C, Patrelli TS, et al. Fertility rate and subsequent pregnancy outcomes after conservative surgical techniques in postpartum hemorrhage: 15 years of literature. Fertil Steril. 2013;99(7): 2097-2107.

18. Barel O, Krakov A, Pansky M, Vaknin Z, Halperin R, Smorgick N. Intrauterine adhesions after hysteroscopic treatment for retained products of conception: what are the risk factors? Fertil Steril. 2015;103(3): 775-779.

19. Sentilhes L, Kayem G, Ambroselli C, et al. Fertility and pregnancy outcomes following conservative treatment for placenta accreta. Hum Reprod. 2010;25(11):2803-2810.

20. Doumouchtsis SK, Arulkumaran S. The morbidly adherent placenta: an overview of management options. Acta Obstet Gynecol Scand. 2010;89(9):1126-1133.

21. Grillo-Ardila CF, Ruiz-Parra AI, Gaitan HG, Rodriguez-Malagon N. Prostaglandins for management of retained placenta. Cochrane Database Syst Rev. 2014;(5):CD010312.
Therapeutics and Clinical Risk Management

\section{Publish your work in this journal}

Therapeutics and Clinical Risk Management is an international, peerreviewed journal of clinical therapeutics and risk management, focusing on concise rapid reporting of clinical studies in all therapeutic areas, outcomes, safety, and programs for the effective, safe, and sustained use of medicines. This journal is indexed on PubMed Central, CAS,
Dovepress

EMBase, Scopus and the Elsevier Bibliographic databases. The manuscript management system is completely online and includes a very quick and fair peer-review system, which is all easy to use. Visit http://www.dovepress.com/testimonials.php to read real quotes from published authors. 